\title{
Human Lens Transmission of Blue Light: A Comparison of Autofluorescence-Based and Direct Spectral Transmission Determination
}

\author{
Adam Elias Broendsted Michael Stormly Hansen Henrik Lund-Andersen \\ Birgit Sander Line Kessel \\ Department of Ophthalmology, Glostrup Hospital, University of Copenhagen, Copenhagen, Denmark
}

\section{Key Words}

Autofluorescence - Human lens - Blue light transmission •

In vivo determination

\begin{abstract}
Purpose: Direct measurement of the transmission of light through the human lens is not possible in vivo unless invasive techniques are used. In the current study, a reliable in vivo estimate of the transmission of blue light through the lens was assessed by comparing an indirect and noninvasive method based on autofluorescence measurements with a direct method. Methods: Total transmission of blue light was measured in human donor lenses using a direct method applicable only in vitro and compared with transmittance estimates made by an in vivo applicable autofluorescence technique. Results: Human lens transmission of blue light decreases with age by $0.7-0.8 \%$ per year at $480 \mathrm{~nm}$. The comparison of methods showed that the autofluorescencebased method correlated significantly with the direct measurements $(R=0.83, p<0.001)$ and acceptable agreement between the two methods was found. Discussion: In conclusion, the human lens transmittance of blue light can be measured reliably in vivo. This enables the possibility to correct for retinal light intensities when studying the mechanisms of
\end{abstract}

the circadian rhythm in clinical studies and related disorders and in addition when working with clinical and experimental methods affected by retinal blue light intensities.

Copyright $\odot 2011$ S. Karger AG, Basel

\section{Introduction}

As the human body ages, the transmission of blue light to the retina decreases due to an increased absorption in the lens of the eye preferentially in the blue spectral region [1]. The decrease in transmission is caused by the accumulation of yellow chromophores acting as an effective filter for blue light causing impaired color vision [2]. The lens proteins are extremely long lived and robust [3] but signs of denaturation are detectable from an early age in the form of insoluble yellow fluorescent protein aggregates [4]. Ultimately, this process may contribute to cataract but it is first and foremost a sign of normal ageing. There are several reasons to measure this decrease in transmission that occurs naturally with age and is accelerated with diseases, e.g. diabetes $[2,5]$.

For instance, in order to compare different age groups, corrections for lens transmission should be made when using ophthalmologic examinations operating in the

Adam Elias Broendsted

Glostrup Hospital, Department of Ophthalmology

Nordre Ringvej 57

DK-2600 Glostrup (Denmark)

Tel. +45 2553 4365, Fax +45 4323 4669, E-Mail adam@eliaz.dk 
short wavelength spectral area [6] or when studying scotopic vision [7, 8]. Measurements of the transmission should also be applied when studying the ageing lens itself and how these changes may affect general health and well-being through alterations in regulation of the circadian rhythm. A newly discovered subset of intrinsically photosensitive retinal ganglion cells, sensitive only to blue light [9], pass information about the external luminance to the suprachiasmatic nucleus acting as the internal clock [10-12]. The age-induced decrease in blue light reduces retinal blue light intensities. Hypothetically, less stimulation of the intrinsically photosensitive retinal ganglion cells may result in a demodulation of the circadian rhythm. On the other hand, the retina is susceptible to blue light phototoxic damage [13-15], which is why there is reason to investigate the protective effects of blue light absorption by the lens.

Various methods to estimate the lens transmission have been described. Most of these are based on the autofluorescence capabilities of the lens $[16,17]$ but in addition, lens transmission can be estimated based on reflected light from the retina, by a psychophysical approach [18] or by measurements of visually evoked potentials [19]. In this methodological study, we have assessed the reliability of an indirect noninvasive method of estimating the transmission of blue light in the human lens using a commercially available instrument based on autofluorescence measurements and compared this method with direct measurements on human donor lenses in vitro.

\section{Materials and Methods}

\section{Human Donor Lenses}

Human donor lenses were provided by the Lions Eye Institute for Transplant and Research, Tampa, Fla., USA. Lenses were received and stored at $5{ }^{\circ} \mathrm{C}$ in Optisol GS transport medium and were used for the experiments within 1 day of arrival and no later than 1 week after death. All lenses showed normal age-related optical changes but did not have any localized opacities in the central part of the lens as such opacities may affect lens autofluorescence measurements [20]. For all measurements, the lenses were placed in $5-\mathrm{mm}$ path length quartz cuvettes containing a neutral, saline solution (in g/l: $\mathrm{NaCl} 8.00, \mathrm{KCl} 0.40, \mathrm{Na}_{2} \mathrm{HPO}_{4}$ 0.10 , glucose 1.00 , Hepes 2.38 , buffered to a $\mathrm{pH}$ of 7.4 using $0.5 \mathrm{M}$ $\mathrm{NaOH})[21]$.

\section{Direct Determination of Spectral Transmission in vitro}

The transmission of the human lenses was measured in the most central part of the lens along the visual axis with the lenses placed with the anterior surface of the lens facing towards the white light source. A broad-band supercontinuum white light la- ser with a beam diameter of $1 \mathrm{~mm}$ was used as the light source (SuperK Blue, Koheras A/S, Denmark). After passing through the cuvette containing the human lens, all the transmitted light was collected by an integrating sphere (FOIS-1, Ocean Optics, The Netherlands) that was coupled to a spectrometer (USB4000, Ocean Optics) by an optical fiber (P600-2-UV-VIS from Ocean Optics). The spectrometer was controlled by a computer program (Spectra Suite, Ocean Optics). This method, employing an integrating sphere, measures the total transmitted light including scattered light (transmission).

Transmission was calculated according to Beer-Lambert's law as the ratio between the intensity of the incident light and the light leaving the material. The formula was rewritten to correct for background light levels that were measured prior to lens transmission measurements. The light measurements provided values for transmission within the spectral region from 400 to $800 \mathrm{~nm}$ at approximately every $0.21 \mathrm{~nm}$. Data were limited to 3 intervals: red (655-665 nm - $\left.T_{660}\right)$, green $\left(525-535 \mathrm{~nm}-T_{530}\right)$ and blue (475-485 $\left.\mathrm{nm}-T_{480}\right)$. The latter was chosen since the absorption peak for melanopsin is located at $480 \mathrm{~nm}$ [22]. These measurements were used as the golden standard when comparing the transmittances found with the indirect method.

\section{Indirect, Autofluorescence-Based Transmittance \\ Measurements}

An autofluorescence profile of the lens was obtained along the visual axis using a commercially available fluorometer (Fluorotron Master, Ocumetrics, Mountain View, Calif., USA). This device projects a beam of blue light in the form of a vertical slit into the eye. At the same time, a detector filtered to allow only fluoresced light is focused on the same point in the eye (fig. 1). The Fluorotron Master was fitted with an anterior segment adapter and records fluorescence in 149 steps along the visual axis of the eye. The excitation wavelength was from 430 to $490 \mathrm{~nm}$ and the fluorescence detection was from 530 to $630 \mathrm{~nm}$. The excitation spectrum is shown in combination with the human spectral transmission (age 18 and 76 years) and the absorption curve for melatonin suppression (fig. 2). Autofluorescence measurements were performed in a dark room with the cuvettes containing the lenses placed in a special build holder that allowed for visual control of the correct positioning of the lens relative to the excitation source. All autofluorescence profiles were recorded along the visual axis in the most central part of the lens. For each lens, the autofluorescence profile was recorded 3 times with the anterior lens surface facing the instrument and 3 scans were recorded with the posterior surface facing the instrument. The raw data were exported and the anterior and posterior lens peaks $\left(P_{\text {Ant }}\right.$ and $\left.P_{\text {Post }}\right)$ were identified for each scan and the mean of the peak value \pm the 3 neighboring measurements were used to calculate the fluorescence intensity of the lens peaks (fig. 3 ).

The autofluorescence-based transmittance $\left(T_{F L}\right)$ can be calculated from $P_{\text {Ant }}$ and $P_{\text {Post }}$ based on Beer-Lambert's law. Due to absorption in the lens $P_{\text {Post }}$ seems lower than $P_{\text {Ant }}$ and the ratio between these is thus the transmittance. However, this requires that the emitted fluorescence intensities in the anterior and posterior part of the lens are equal or that the intrinsic peak ratio equals 1 . Congruently to previous work $[23,24]$, recordings of fluorescence profiles of the lens with the anterior surface facing the fluorometer (similar to in vivo measurements) and with the posterior surface facing the fluorometer (opposite to in vivo measurements) 
Fig. 1. A schematic of the mechanism behind the Fluorotron Master. The light source filtered to allow only blue light to pass excites the lens by a vertical slit; note how the intensity of the transmitted blue light is reduced by absorption through the lens. The detector filtered to allow only fluoresced light records the emitted fluorescence from the exact point excited by the light source, which is repeated 149 times along the visual axis. The diagram beneath the eye shows a typical lens autofluorescence profile. Inside the enlargement of the lens, the absorption of photons (A) by the lens proteins resulting in fluorescence $(\mathrm{F})$ is illustrated by a Jablonski diagram.

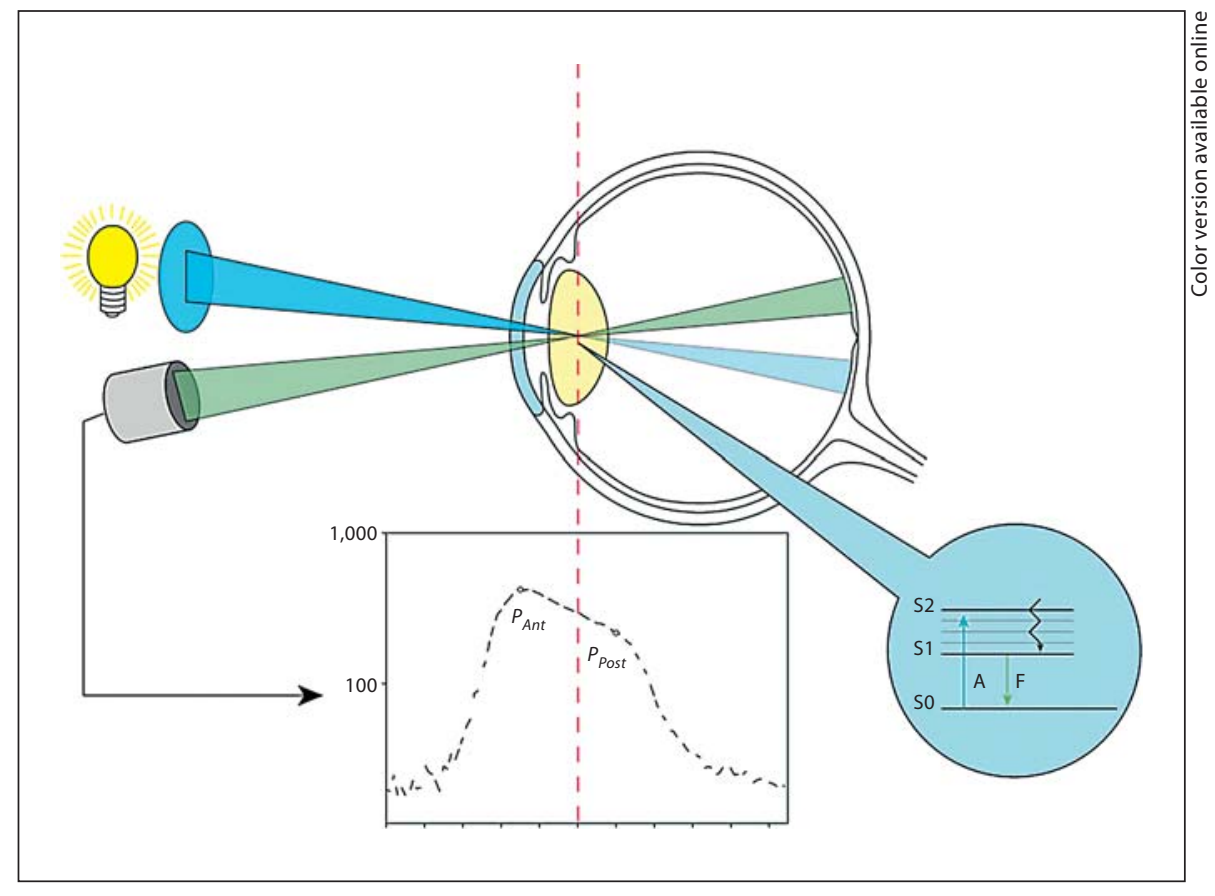

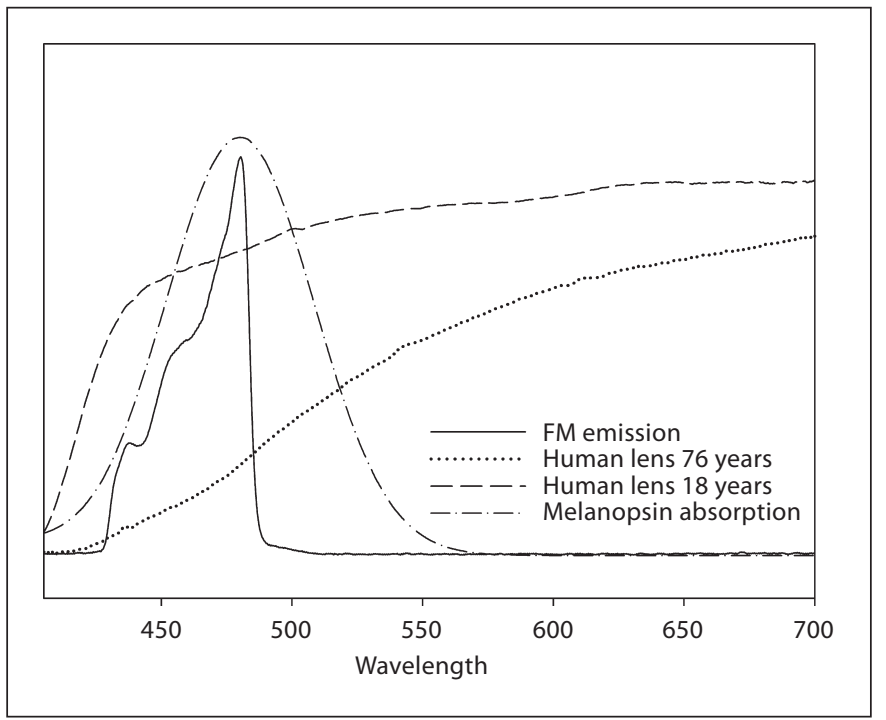

Fig. 2. The Fluorotron Master (FM) excitation spectrum in combination with the spectral absorption curve for melanopsin [18] illustrating that these peak in the same spectral area. This also shows that the transmittance based on autofluorescence measured with the Fluorotron Master is specific for blue light. Shown is also the spectral transmittance of a young and elderly human lens as an example of the reduced blue light transmission with age.

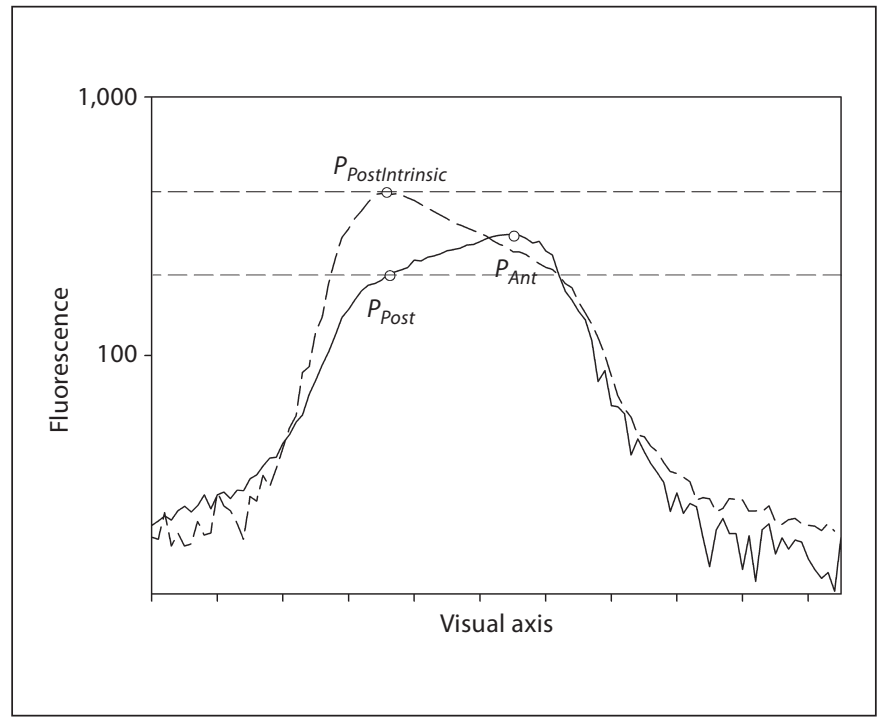

Fig. 3. An autofluorescence scan through the anterior chamber of the eye. The marked peaks represent the anterior and posterior lens peaks $\left(P_{\text {Ant }}\right.$ and $\left.P_{\text {Post }}\right)$. The dashed line shows the same lens when recorded with the posterior surface facing the Fluorotron Master illustrating the difference between the measured posterior peak and the actual fluorescence in the posterior lens segment $\left(P_{\text {PostIntrinsic }}\right)$. The transmittance can be calculated as the ratio between $P_{\text {Post }}$ and $P_{\text {PostIntrinsic }}$. As the latter cannot be measured in vivo, it was empirically estimated from $P_{\text {Ant }}$. 
Table 1. Data (mean $\pm \mathrm{SD}$ ) used for calculating the transmittance as well as the direct transmission measurements

\begin{tabular}{lclllllllll}
\hline Age group & $\mathrm{n}$ & Age & $P_{\text {Ant }}$ & $P_{\text {Post }}$ & $P_{\text {PostIntrinsic }}$ & Corr & $T_{F L}$ & $T_{480}$ & $T_{530}$ & $T_{660}$ \\
\hline $18-39$ & 7 & $23.1 \pm 5.1$ & $131 \pm 26$ & $97 \pm 26$ & $144 \pm 27$ & $1.10 \pm 0.03$ & $0.71 \pm 0.09$ & $0.75 \pm 0.04$ & $0.88 \pm 0.03$ & $0.97 \pm 0.02$ \\
\hline $40-59$ & 4 & $50.3 \pm 5.6$ & $607 \pm 218$ & $256 \pm 46$ & $729 \pm 226$ & $1.20 \pm 0.13$ & $0.38 \pm 0.11$ & $0.42 \pm 0.09$ & $0.69 \pm 0.06$ & $0.97 \pm 0.02$ \\
\hline $60-80$ & 21 & $68.9 \pm 5.6$ & $533 \pm 178$ & $241 \pm 74$ & $697 \pm 243$ & $1.31 \pm 0.39$ & $0.35 \pm 0.07$ & $0.35 \pm 0.12$ & $0.62 \pm 0.12$ & $0.93 \pm 0.10$
\end{tabular}

To show the age variation the lenses have been split into three age groups: $18-39$ years of age, $40-59$ years of age and 60-80 years of age. $P_{\text {Ant }}, P_{\text {Post }}$ and $P_{\text {PostIntrinsic }}$ show the average measured values for these parameters for the three age groups. $\operatorname{Corr}(C)$ or the intrinsic peak ratio show the statistically established $P_{\text {PostIntrinsic }}$ $P_{\text {Post }}$ ratio used to calculate the transmittance. $T_{F L}$ and $T_{480}$ show

the average values for the autofluorescence-based method and the direct spectral measurement. Lastly, since the direct spectral determination of the transmission provided transmission values at a wide spectral area, values are shown for green $\left(T_{530}\right)$ and red $\left(T_{660}\right)$ light also.

Table 2. The mean autofluorescence-based transmittance measurement $\left(T_{F L}\right)$ and direct determined transmission of blue light

\begin{tabular}{lllll}
\hline Transmission & Mean & Slope & Intercept & $\mathrm{R}^{2}$ correlated to $T_{F L}$ \\
\hline$T_{F L}(95 \% \mathrm{CI})$ & $0.43(0.10$ to 0.76$)$ & -0.007 per year $(-0.009$ to -0.006$)$ & $0.84(0.74$ to 0.94$)$ & - \\
$T_{480}(95 \% \mathrm{CI})$ & $0.45(0.07$ to 0.83$)$ & -0.008 per year $(-0.01$ to -0.006$)$ & $0.90(0.78$ to 1.02$)$ & $(0.71)$ \\
$T_{530}(95 \% \mathrm{CI})$ & $0.69(0.43$ to 0.95$)$ & -0.005 per year $(-0.006$ to -0.003$)$ & $0.96(0.87$ to 1.06$)$ & $(0.65)$ \\
$T_{660}(95 \% \mathrm{CI})$ & $0.95(0.87$ to 1.02$)$ & -0.001 per year $(-0.001$ to -0.000$)$ & $0.99(0.95$ to 1.03$)$ & $(0.17)$ \\
\hline
\end{tabular}

The direct determined transmission of blue light $\left(T_{480}\right)$ and the indirect method $\left(T_{F L}\right)$ are neither statistically different with regard to mean, intercept nor slope $(\mathrm{p}>0.1)$. $\mathrm{R}^{2}$ shows the coefficient of determination when the method is compared to $T_{F L}$. The table also shows the mean transmission and the age correlation of green and red light. The $\mathrm{R}^{2}$ values are put in parentheses because the comparison of $T_{F L}$ with these values is somewhat futile since we can assume that $T_{F L}$ will provide transmittance values in the blue spectral region given the excitation spectrum. revealed that the intrinsic peak ratio differed from 1 and that a correction was needed. Since the transmittance could not simply be calculated as the ratio between $P_{\text {Post }}$ and $P_{\text {Ant }}$, the transmittance was calculated as the ratio between $P_{\text {Post }}$ and the intensity of the emitted fluorescence from the posterior lens segment (PostIntrinsic).

$$
T_{F L}=\frac{P_{\text {Post }}}{P_{\text {Postlntrinsic }}}
$$

$P_{\text {PostIntrinsic }}$ is not measurable in vivo and for the method to be useful in clinical settings it had to be estimated from $P_{\text {Ant }}$ using the intrinsic peak ratio, i.e. the ratio between the $P_{\text {PostIntrinsic }}$ and $P_{\text {Ant }}(C)$ established statistically from the autofluorescence scans recorded for all the human donor lenses included in the study.

$$
C=\frac{P_{\text {PostIntrinsic }}}{P_{\text {Ant }}} \Leftrightarrow P_{\text {PostIntrinsic }}=P_{\text {Ant }} C
$$

\section{Statistical Methods}

Statistical analyses were performed using Sigmastat v. 3.5, Systat Software, Inc., USA and for all tests a significance level of 0.05 was chosen and variance was described as $95 \%$ confidence intervals $(95 \% \mathrm{CI})$. On average, the methods were compared by a paired $t$ test. The age relation was tested with linear regression analysis and the produced slopes were tested for parallelism [25]. The two methods were also correlated and the coefficient of determination was assessed. Finally a comparative plot was constructed to consider the agreement between the two methods [26].

\section{Results}

A total of 32 lenses from 21 donors were available for direct determination of spectral transmission as well as regular transmittance indirectly measured with the fluorometer. The average age of the lenses was 56.6 years (from 18 to 80 years; table 1 ).

Direct Determination of Spectral Transmission in vivo

The average transmission of blue light (475-485 nm) was 0.45 with a considerable range (95\% CI 0.066-0.826) 


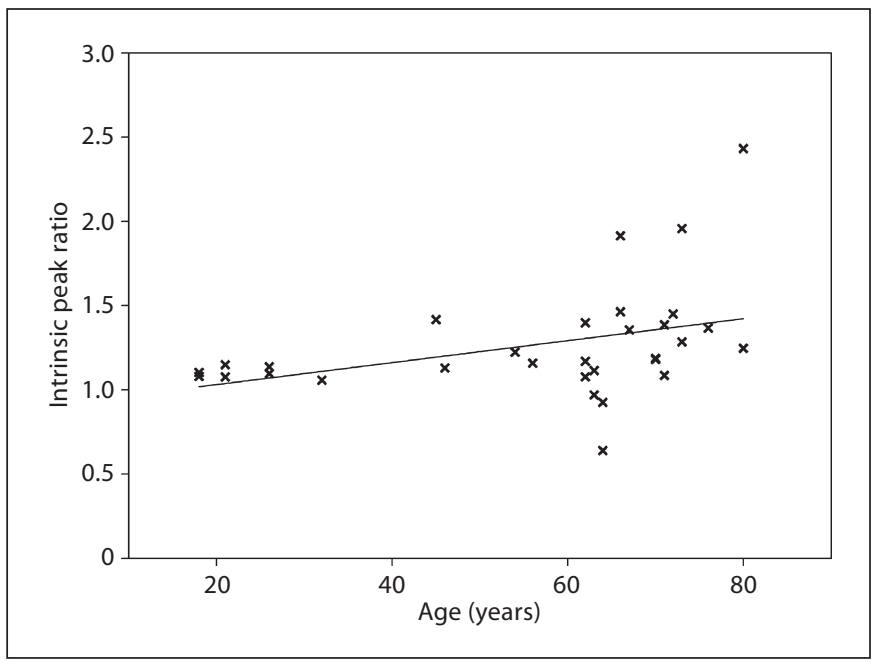

Fig. 4. The $P_{\text {Ant }} / P_{\text {PostIntrinsic }}$ ratio related to age. This correlation was used as an age-dependent correction factor $(C)$ when the blue light transmittance based on autofluorescence measurements of the lens was calculated thus creating an age-corrected blue light transmittance value. due to a significant decrease in transmission with age $(\mathrm{R}=0.81, \mathrm{p}<0.001)$

$$
T=0.89-0.0080 \cdot \text { Age (years) }
$$

Consequently, the average transmission of blue light for an 18-year-old lens was 0.75 , whereas the blue light transmission for an 80 -year-old lens was 0.26 or approximately one third of the 18-year-old (table 2).

\section{Intrinsic Peak Ratio (C)}

The average intrinsic peak ratio $\left(P_{\text {PostIntrinsic }} / P_{\text {Ant }}=C\right)$ was 1.27 (95\% CI $0.62-1.91)$ but strongly dependent on age $(\mathrm{R}=0.39, \mathrm{p}=0.027)$ meaning that more fluorescence was emitted from an 80-year-old posterior lens segment than from an 18-year-old posterior lens segment relative to the fluorescence emitted from the anterior segment (fig. 4):

$$
C=0.90+0.0065 \cdot \text { Age (years) }
$$

Equation 4 was incorporated into equation 1 according to equation 2 producing equation 5 :

$$
T_{F L}=\frac{P_{\text {Post }}}{P_{\text {Ant }}(0.90+0.0065 \cdot \text { Age }(\text { years }))}
$$

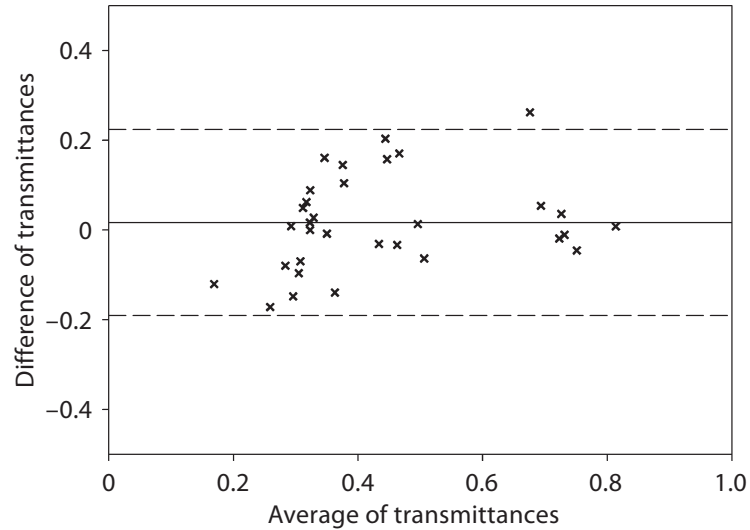

Fig. 5. A Bland-Altman plot where differences of measurements are plotted against the means of the measurements. The dashed lines represent $95 \% \mathrm{CI}$. The plot shows the calculated and corrected autofluorescence-based transmittance $\left(T_{F L}\right)$ compared to the white light transmittance at $480 \mathrm{~nm}\left(T_{W L}\right)$. It shows that the mean difference between the two methods is close to $0(0.0208$, SD 0.106$)$ proving a good mean agreement. The limits of agreement were -0.19 to 0.23 . Consequently the autofluorescencebased method is good when dealing with a large data set.

Indirect Measurement of Regular Lens Transmittance in vitro Using Autofluorescence-Based Method

The average transmittance was $0.43(95 \%$ CI $0.10-$ $0.76)$ but a significant decrease with age was found ( $\mathrm{p}<$ $0.001, \mathrm{R}=0.85)$ :

$$
T_{F L}=0.841-0.007 \cdot \text { Age (years) }
$$

The 95\% CI for the intercept was 0.74-0.94, and -0.0089 to -0.0057 for the slope. The analysis showed that an 18 -year-old's transmittance is reduced by $2 / 3$ at the age of 80 .

\section{Comparison of Direct and Indirect Measurements of \\ Lens Transmission}

On average, the difference between $T_{480}$ and $T_{F L}$ was less than 3\%. Lens transmission measurements obtained using the golden standard and the fluorescence-based method were not statistically different when analyzed with the paired t test $(p=0.275)$ and neither was a significant difference found between the slopes produced by the linear regression analysis $(\mathrm{p}>0.1)$. A significant correlation between the two methods was found $(R=0.84$, $\mathrm{p}<0.0001)$ as well as a high coefficient of determination $\left(\mathrm{R}^{2}=0.71\right.$; table 2$)$. The Bland-Altman comparative anal- 
ysis of $T_{F L}$ and $T_{480}$ showed that the average difference between the two methods was close to $0(0.021)$. The limits of agreement were from -0.19 to 0.23 , which means that at least $95 \%$ of the found differences between measurements were located within the interval. There was no tendency in the distribution of data in the Bland-Altman plot as the data were randomly distributed within the limits of agreement (fig. 5).

\section{Discussion}

The aim of this study was to investigate the possibility of estimating the lens transmission in vivo using a noninvasive method based on lens autofluorescence measurements with a commercially available instrument. This indirect determination of the regular transmittance was compared to a direct determination of the total spectral transmission at $480 \mathrm{~nm}$. Our results showed a good agreement between the two methods demonstrating that the blue light transmission can be assessed in vivo. A strong correlation between age and loss of blue light transmission was found in this study similar to previous work [16]. Furthermore, an age-dependent correction factor was found. However, this correction is specific for these data (healthy subjects) and precautions should be taken in the application of this method on e.g. a diabetic or cataractous patient group. The study showed that the transmission at $480 \mathrm{~nm}$ was reduced by $2 / 3$ at the age of 80 .
An objective, quantitative method for measuring the blue light transmission is very relevant. Hypothetically, decreasing lens transmission may, through a reduction of retinal blue light intensities, impair the entrainment of the circadian rhythm and elderly who have a proven low transmission $[4,27]$ have been shown to suffer more frequently from sleep disorders, which may be caused by demodulation of the circadian rhythm or even phase shift [28]. Studies involving cataract patients have shown an improvement in sleeping patterns after cataract extraction indicating an improvement in circadian functionality [29]. Studies on this topic and the potential harm of blue light could benefit from measurement of the human lens transmission so that retinal light intensities may be assessed. A fast and reliable method for measuring the blue light transmission through the human lens is thus relevant when working with clinical as well as experimental methods affected by retinal blue light intensities and/or working with subjects of different age groups. However, a degree of cooperation is required from subjects, which is why other methods [19] should be applied on very young subjects.

In conclusion, we have shown that the transmission of blue light through the human lens can be assessed in vivo using an autofluorescence-based technique and that this method has a wide range of relevant application possibilities.

\section{References}

1 Kessel L, Lundeman JH, Herbst K, Andersen TV, Larsen M: Age-related changes in the transmission properties of the human lens and their relevance to circadian entrainment. J Cataract Refract Surg 2010;36:308312.

$\checkmark 2$ Kessel L, Alsing A, Larsen M: Diabetic versus non-diabetic colour vision after cataract surgery. Br J Ophthalmol 1999;83:1042-1045.

$\checkmark 3$ Lynnerup N, Kjeldsen H, Heegaard S, Jacobsen C, Heinemeier J: Radiocarbon dating of the human eye lens crystallines reveal proteins without carbon turnover throughout life. PLoS One 2008;3:e1529.

$\checkmark 4$ Lerman S, Borkman R: Spectroscopic evaluation and classification of the normal, aging and cataractous lens. Ophthalmic Res 1976; 8:335-353.
5 van Best JA, Vrij L, Oosterhuis JA: Lens transmission of blue-green light in diabetic patients as measured by autofluorophotometry. Invest Ophthalmol Vis Sci 1985;26:532536.

6 Teesalu P, Airaksinen PJ, Tuulonen A, Nieminen $\mathrm{H}$, Alanko $\mathrm{H}$ : Fluorometry of the crystalline lens for correcting blue-on-yellow perimetry results. Invest Ophthalmol Vis Sci 1997;38:697-703.

7 Schefrin BE, Hauser M, Werner JS: Evidence against age-related enlargements of ganglion cell receptive field centers under scotopic conditions. Vision Res 2004;44:423-428.

$>8$ Werner JS: Night vision in the elderly: consequences for seeing through a 'blue filtering' intraocular lens. Br J Ophthalmol 2005;89: 1518-1521.

$\checkmark 9$ Thapan K, Arendt J, Skene DJ: An action spectrum for melatonin suppression: evidence for a novel non-rod, non-cone photoreceptor system in humans. J Physiol 2001; 535:261-267.
10 Hannibal J, Hindersson P, Knudsen SM, Georg B, Fahrenkrug J: The photopigment melanopsin is exclusively present in pituitary adenylate cyclase-activating polypeptide-containing retinal ganglion cells of the retinohypothalamic tract. J Neurosci 2002; 22:RC191

- 11 Hattar S, Liao HW, Takao M, Berson DM, Yau KW: Melanopsin-containing retinal ganglion cells: architecture, projections, and intrinsic photosensitivity. Science 2002;295: 1065-1070.

12 Berson DM: Strange vision: ganglion cells as circadian photoreceptors. Trends Neurosci 2003;26:314-320.

13 Werner IS, Steele VG, Pfoff DS: Loss of human photoreceptor sensitivity associated with chronic exposure to ultraviolet radiation. Ophthalmology 1989;96:1552-1558.

14 Ham WT Jr, Mueller HA, Sliney DH: Retinal sensitivity to damage from short wavelength light. Nature 1976;260:153-155. 
15 Boulton M, Rozanowska M, Rozanowski B: Retinal photodamage. J Photochem Photobiol B 2001;64:144-161.

-16 Zeimer RC, Lim HK, Ogura Y: Evaluation of an objective method for the in vivo measurement of changes in light transmittance of the human crystalline lens. Exp Eye Res 1987;45: 969-976.

-17 van Best JA, Tsoi EW, Boot JP, Oosterhuis JA: In vivo assessment of lens transmission for blue-green light by autofluorescence measurement. Ophthalmic Res 1985;17:90-95.

18 Delori FC, Burns SA: Fundus reflectance and the measurement of crystalline lens density. J Opt Soc Am A Opt Image Sci Vis 1996;13: 215-226.

19 Werner JS: Development of scotopic sensitivity and the absorption spectrum of the human ocular media. J Opt Soc Am 1982;72: 247-258.
20 Siik S, Chylack LT Jr, Friend J, Wolfe J, Teikari J, Nieminen H, Airaksinen PJ: Lens autofluorescence and light scatter in relation to the lens opacities classification system, LOCS III. Acta Ophthalmol Scand 1999;77: 509-514.

21 Glasser A, Campbell MCW: Biometric, optical and physical changes in the isolated human crystalline lens with age in relation to presbyopia. Vision Res 1999;39:1991-2015.

22 Brainard GC, Sliney D, Hanifin JP, Glickman G, Byrne B, Greeson JM, Jasser S, Gerner E, Rollag MD: Sensitivity of the human circadian system to short-wavelength (420nm) light. J Biol Rhythms 2008;23:379-386.

23 Larsen M, Lund-Andersen H: Lens fluorometry: light-attenuation effects and estimation of total lens transmittance. Graefes Arch Clin Exp Ophthalmol 1991;229:363-370.

24 Jacobs R, Krohn DL: Fluorescence intensity profile of human lens sections. Invest Ophthalmol Vis Sci 1981;20:117-120.
25 Kleinbaum D, Kupper L, Muller K, Nizam A: Applied Regression Analysis and Other Multivariable Methods. Pacific Grove, Duxbury Press, 1998

26 Bland JM, Altman DG: Statistical methods for assessing agreement between two methods of clinical measurement. Lancet 1986;i:307-310.

$>27$ Kessel L, Lundeman JH, Herbst K, Andersen TV, Larsen M: Age-related changes in the transmission properties of the human lens and their relevance to circadian entrainment. J Cataract Refract Surg 2010;36:308312.

28 Asplund R: Sleep, health and visual impairment in the elderly. Arch Gerontol Geriatr 2000;30:7-15.

29 Asplund R, Lindblad BE: Sleep and sleepiness 1 and 9 months after cataract surgery. Arch Gerontol Geriatr 2004;38:69-75. 